\title{
LA RADIO EN EL ECOSISTEMA MEDIÁTICO DEL SIGLO XXI: ESTUDIO DE CASO EN PORTUGAL
}

\section{RADIO IN THE $21^{\text {st }}$ CENTURY MEDIA ECOSYSTEM: CASE STUDY IN PORTUGAL}

https://doi.org/10.33732/ixc/10/03Laradi

\section{João Canavilhas}

Universidade da Beira Interior/LABCOM

jc@ubi.pt

http://orcid.org/0000-0002-2394-5264 
Resumen: Desde que aparece la televisión, la muerte de la radio se anuncia como cercana. A pesar de todas las amenazas, el medio continúa vivo e, incluso, gana nuevos públicos en un escenario hostil para un medio centenario. La realidad muestra que la radio transformó sus debilidades en fuerzas y se adecuó al nuevo ecosistema de una forma más rápida que cualquier otro medio. Las razones de esta veloz y exitosa adaptación están relacionadas con la simplicidad del soporte y con el lenguaje unisensorial usado, pero también con una capacidad inaudita para el reajuste a nuevas situaciones. En este trabajo se identifican los rasgos que hacen de la radio una superviviente y se analiza la forma como se adaptó al nuevo ecosistema, ilustrando cada caso con ejemplos de programas radiofónicos portugueses.

Palabras clave: radio; Internet; ecosistema mediático; nuevos medios; digital.

\begin{abstract}
Since the appearance of television, the death of the radio has been expected. However, despite all the threats it is still alive and has seen an increase in audiences in a hostile scenario for a centennial media. Reality shows that the radio has transformed its weaknesses into strengths and has adapted to the new ecosystem faster than any other media. The reasons for this quick and successful adaptation are related to the simplicity of the support and to its unisensorial language, but also to an unprecedented capacity of adaptation to new situations. This work identifies the characteristics that makes radio a survivor and analyzes the way in which the radio adapted itself to the new ecosystem, illustrating each case with examples from Portuguese radios.
\end{abstract}

Keywords: Radio; Internet; Media Ecosystem;

New Media; Digital. 


\section{Introducción}

Tal como ocurre en los ecosistemas biológicos, donde la entrada de una nueva especie provoca desequilibrios inmediatos, la aparición de un nuevo medio de comunicación altera el ecosistema mediático con efectos visibles a nivel de los factores intermediáticos, contextuales y tecnoambientales (Canavilhas, 2011). Desde luego porque se introduce un nuevo elemento en un espacio equilibrado, pero también porque aumenta la complejidad del sistema al añadir diversidad y sofisticación. Fue lo que ocurrió a principios del siglo XXI con la expansión de la World Wide Web: el impacto de la Web en los medios de comunicación tradicionales se sintió con diferentes intensidades, pero todos sufrieron alguna erosión con la aparición de un nuevo elemento.

En el caso específico de la radio, el primer gran impacto causado por otro medio ocurrió con la popularización de la televisión, en los años 60 del siglo pasado. Aunque perdió la preferencia de las audiencias en las zonas más desarrolladas del globo, la radio mantuvo una importante cuota de audiencia mundial y, a inicios del nuevo siglo, aún era el medio con mayor penetración a escala global (Ahonen, 2011). Con la emergencia de la Web, la amenaza a los viejos medios se repitió, pero si la prensa se ha sumergido en una crisis y la televisión se resintió, la radio resistió.

Esta resistencia se debe a un conjunto de características del propio medio que le han permitido adaptarse a las novedades. Este ajuste, conocido como mediamorfosis (Fidler, 1997) o remediación (Bolter \& Grusin, 1999), ha propiciado la evolución del medio radiofónico con relación a determinados aspectos de Internet $y$, en particular, de la Web.

Si el contenido de un nuevo medio es siempre un medio anterior (McLuhan, 1969), lo contrario también puede ocurrir y es justo lo que pasó con la radio en su proceso de adaptación al nuevo ecosistema. Las razones de este rápido y exitoso proceso están relacionadas con una capacidad inaudita del medio para integrarse en nuevas situaciones. A modo de ejemplo se puede analizar la performance de las radios en los premios anuales de ciberperiodismo asignados en Portugal. En 11 ediciones, Rádio Renascença ganó siete veces el premio Excelencia, descrito por la organización como un sitio que «[...] cumpra com sucesso as suas funções editoriais, sirva efetivamente a sua audiência, maximize a exploração das características da Internet e represente os mais elevados standards jornalísticos. Os sites serão julgados na excelência do 
conteúdo, interatividade, multimedialidade, design, usabilidade e ferramentas de comunidade.» ${ }^{1}$ (Obciber, 2019).

El hecho de que un medio amenazado desde los años 80 tenga la capacidad de amoldarse a las nuevas realidades y se destaque en el nuevo ecosistema mediático justifica un análisis buscando entender cómo ha podido la radio transformar sus fragilidades en oportunidades.

En parte, el éxito puede explicarse por la existencia de un fuerte vínculo entre las versiones tradicionales y online del medio al comprobarse «[...] um aumento da utilização de rádio na internet à medida que aumenta a frequência de audição de rádio FM [...]»² (Portela, 2015: 211). El autor también destaca que «[...] nunca antes à rádio se ofereceram tantas oportunidades tecnológicas de estabelecer pontes sonoras (e, agora, também multimediáticas) com as suas audiências» ${ }^{3}$ (Portela, 2015: 103) por lo que interesa saber cuáles son las características que permiten a la radio sobrevivir en el actual ecosistema mediático.

\section{Un ecosistema en cambio: remediación y mediamorfosis}

En un ecosistema biológico existen varios tipos de consumidores - primarios, secundarios y terciarios - que se organizan en una cadena jerárquica en la que los consumidores primarios se alimentan de los productores y los siguientes se alimentan siempre del consumidor anterior.

Trazando una analogía con el ecosistema mediático, podemos identificar una repetición de esta tendencia cuando McLuhan (1969) dice que el contenido de un medio es siempre otro medio; es decir, cada nuevo medio se alimenta del anterior. En realidad, la radio sonorizó la palabra de la prensa, la televisión añadió imagen en movimiento al sonido de la radio, la Web juntó interactividad al contenido multimedia de la televisión y, más recientemente, los smartphones agregaron el consumo personalizado y en movilidad.

En esta misma corriente, Levinson (1997: 104) introduce el concepto de remediación, definiéndolo como «the process by which new media technologies

1 «[...] sirva efectivamente a su audiencia, maximice la explotación de las características de Internet y represente los más altos estándares periodísticos. Los sitios serán juzgados en la excelencia del contenido, interactividad, multimedialidad, diseño, usabilidad y herramientas de comunidad».

$2 \ll[. .$.$] un aumento de la utilización de la radio en internet a medida que aumenta la frecuencia$ de audición de radio FM [...]».

3 «[...] jamás se había ofrecido a la radio tantas oportunidades tecnológicas para establecer puentes de sonido (y ahora también multimedia) con sus audiencias [...]». 
improve upon or remedy prior technologies» ${ }^{4}$. Dos otros autores, Bolter \& Grusin (1999: 273) desarrollan este concepto y hablan de un proceso de remediación descrito como «the formal logic by which new media refashion prior media forms $»^{5}$. Aunque las definiciones parecen similares, Bolter \& Grusin, (1999: 59) subrayan una diferencia importante al considerar que este proceso ocurre en los dos sentidos: «older media can also refashion newer ones. Newer media do not necessarily supersede older media because the process of reform and refashioning is mutual» 6 . A este respecto, Canavilhas (2012) habla de remediación inversa, proponiendo esta denominación para dejar claro el proceso en el que los nuevos medios influyen igualmente en los medios anteriores.

En el caso de nuestro objeto de estudio - la radio - esta remediación inversa es visible en los programas televisivos genéricamente denominados antena abierta. En el proceso normal, los debates televisivos añadieron imagen a los debates radiofónicos, pero en un proceso de inverso, la televisión recuperó el formato de los foros radiofónicos, pasando a tener programas donde los telespectadores participan a través de una llamada telefónica usando sólo el sonido, tal como ocurre en la radio. Se trata de una limitación técnica inicial, pero que se ha transformado en un formato televisivo.

Este proceso de remediación puede ocurrir con diferentes intensidades. En la versión más simple, el viejo medio es únicamente destacado y representado en el nuevo, manteniéndose un vínculo fuerte y un diálogo permanente entre ambos. Desde este punto, cuanto más transparente sea la interfaz, mejor será la relación entre el consumidor y el contenido porque los cambios son menores. La versión pdf para tablets, por ejemplo, es una mejora del formato tradicional al permitir una distribución inmediata, pero mantiene las características fundamentales del periódico en papel. En un nivel más avanzado, el medio digital puede presentar una remodelación completa (refashion) del antiguo medio, aunque su presencia sigue estando presente. Es lo que ocurre en los programas televisivos de entrevistas, en los que se añade imagen a un formato típico de la radio.

Fidler (1997: 22-23) analiza el proceso de sucesión mediática llamándole mediamorfosis. El autor define este proceso como «the transformation of a communication medium, usually brought about the complex interplay of perceived

\footnotetext{
4 «El proceso mediante el cual las nuevas tecnologías de los medios mejoran o remedian tecnologías anteriores».

5 «La lógica formal mediante la cual los nuevos medios remodelan formas de medios anteriores».

6 «Los medios más antiguos también pueden remodelar los más nuevos. Los medios más nuevos no reemplazan necesariamente a los medios más antiguos porque el proceso de reforma y remodelación es mutuo».
} 
needs, competitive and political pressures, and social and technological innovations ${ }^{7}$. A partir de esta idea, Fidler (1997) identifica seis principios que subyacen a la mediamorfosis: 1) coevolución (todos los medios coexisten y evolucionan en paralelo, aunque a diferentes velocidades); 2) metamorfosis (ningún medio surge de forma espontánea e independiente, sino por la transformación de un medio preexistente); 3) propagación (los nuevos medios mimetizan las características dominantes de los medios anteriores a través de códigos llamados lenguajes); 4) supervivencia (todos los medios buscan adaptarse y evolucionar para sobrevivir en el ecosistema); 5) oportunidad y necesidad (el éxito de los nuevos medios no depende únicamente de los méritos tecnológicos, sino también de razones económicas y sociales que pueden determinar su éxito o fracaso); 6) adopción tardía (las tecnologías asociadas a nuevos medios demoran entre 20 y 30 años en ser un éxito comercial).

Aunque podemos encuadrar la radio en los diversos puntos, en este caso nos interesa el 4, ya que este medio es el mejor ejemplo de supervivencia al adaptarse a los nuevos medios a través del perfeccionamiento en sus características tradicionales y de la remediación de los medios posteriores.

Aunque subsisten algunas diferencias entre los conceptos de remediación y de mediamorfosis, hay dos rasgos comunes que pueden ser confirmados en cualquier fase de evolución en el ecosistema: a) los nuevos medios no nacen espontáneamente: cada nuevo medio evoluciona a partir de uno o de varios medios anteriores, manteniendo con ellos una conexión; b) los viejos medios buscan aprovechar algunas características de los nuevos medios, intentando así mantener su rol en el ecosistema. Es en esta dialéctica que funciona la lucha por la supervivencia en un ambiente que se vuelve adverso por la llegada de una nueva especie mediática.

\section{Radio amenazada: de la televisión a la Web}

A pesar de que el período experimental de la radio se localiza a finales del siglo XIX, su aparición como medio de comunicación se sitúa en el período entre las dos grandes guerras mundiales (Jeanneney, 1996). En la II Guerra Mundial, la radio ya era un medio de comunicación con tasas de penetración relevantes y por eso fue ampliamente usada como arma de guerra en varios escenarios bélicos. La Alemania nazi esparcía las ideas del Mein Kampf a través de este medio, llegando a distribuir a su población un tipo de radio que sólo sintonizaba las emisoras del régimen. En la ocupación de China por parte de Japón, los

7 «La transformación de un medio de comunicación, generalmente provocada por la compleja interacción de necesidades percibidas, presiones competitivas y políticas e innovaciones sociales y tecnológicas 
invasores instalaban altavoces en lugares públicos para transmitir sus mensajes políticos.

En este período ocurrieron manipulaciones masivas con arreglo a la radio en muchos otros países, incluyendo España, cuyo régimen instaló en 1937 un emisor que permitía hacer llegar los mensajes nacionalistas a todo el territorio (Jeanneney, 1996). En el lado de los Aliados, la radio fue igualmente usada, sobre todo la inglesa BBC con los mensajes de Winston Churchill. Las radios americanas Voz de América y American Forces Network fueron igualmente dos referencias para el público europeo de esa época.

El sustancial papel desempeñado por la radio en este conflicto mundial le permitió ganar un lugar especial en el menú mediático de los ciudadanos, más aún porque durante la II Guerra la prensa se hundió en una crisis por la dificultad en conseguir papel y por el bloqueo militar a los circuitos de distribución, situación que redujo su espectro de influencia. La radio ha sustituido a la prensa porque su proceso de distribución y consumo es más simple y accesible. En realidad, hasta quien no sabía leer pasó a tener acceso a la información gracias a la radio.

En las tres décadas de la posguerra - 40, 50 y 60-, la radio se benefició de varias innovaciones tecnológicas que le permitieron cimentar su lugar en el ecosistema mediático. La aparición de la FM (frecuencia modulada) mejoró la calidad sonora de los contenidos, los grabadores permitieron la sustitución de las bobinas, la miniaturización de los receptores hizo el consumo más móvil y la producción en masa permitió una reducción de su precio que aumentó la velocidad en su popularización. En paralelo, las estaciones de radios se multiplicaron y segmentaron, surgiendo los canales temáticos y, ya en los años 70 e inicios de los 80, las llamadas emisoras libres o piratas crearon una audiencia de proximidad gracias a las emisiones locales. Pero después de esta época dorada, la radio vendría a atravesar su primera gran crisis con el crecimiento de la televisión.

\subsection{Primer shock: la televisión}

El fenómeno televisivo empieza a ganar dimensión pública en los años 30 del siglo XX, pero en esa época la radio estaba en el centro de la atención. La mayor exigencia técnica de la televisión en términos de emisión/recepción fue un obstáculo para su rápida expansión y solo en la posguerra el fenómeno comenzó a despertar verdaderamente el interés del público generalista. En 1953, la transmisión de la coronación de Isabel II fue el primer momento en que las audiencias televisivas inglesas sobrepasaron las radiofónicas (Jeanneney, 1996). Es precisamente en la difusión de estos grandes eventos donde se asienta el papel 
protagónico que la televisión va a ocupar en el ecosistema. Un poco por todo el mundo, en los años 60 comienzan las emisiones regulares de canales televisivos. El asesinato del presidente estadounidense John Kennedy, la Guerra de Vietnam y el deporte, sobre todo la transmisión de grandes eventos deportivos como los campeonatos mundiales de fútbol o los Juegos Olímpicos, vendrían a cimentar el lugar de la televisión.

Con las emisiones en color, la reducción de precios de los televisores y la aparición de los satélites, la televisión se convierte en un fenómeno global y, a partir de los años 80 , ya es el medio socialmente más destacado. En este escenario, la radio sufre su primer ataque revelando muchas dificultades para resistir el atractivo de la imagen en movimiento ofrecida por la televisión.

En los años 90 del siglo XX, la televisión es cada vez más ágil y trata de usar igualmente una de las fuerzas de la radio: el directo. Durante la Guerra del Golfo, la norteamericana CNN mostró todo el potencial de la televisión en un terreno hasta entonces dominado por la radio y ganó definitivamente el liderazgo de las audiencias en los países desarrollados. Con la digitalización, la televisión mejoró la calidad y aumentó la velocidad de producción y distribución de contenidos a escala global, afirmándose como el medio más poderoso. A partir de este momento, la televisión se segmentó en decenas de modelos económicos, en cientos de formas para llegar al público, en miles de canales especializados y en millones de formatos, todo ello, en aras de mantener su supremacía en el ecosistema en los países desarrollados. La radio quedó reducida a las audiencias de proximidad (geográfica o temática) y a los oyentes en los automóviles.

\subsection{Segundo shock: la Web}

Si bien el origen de la Web se ubica a finales de los años 60, con la aparición de ARPANet, una red de ordenadores militares, fue en 1989 cuando Tim Berner-Lee lanzó la Web y al año siguiente cuando desarrolló todas las herramientas necesarias para su funcionamiento. Con la aparición de los navegadores y la comercialización de los servicios de acceso a la Web, a partir de la mitad de la década comienzan a aparecer espacios comerciales, como Amazon o eBay, ambos creados en 1995. El éxito del nuevo medio llevó al aumento de la información disponible y el surgimiento del motor Altavista que, en el mismo año, vino a ayudar en la función de organizar contenidos y facilitar las búsquedas.

En los años siguientes, la Web entró en un proceso de crecimiento gracias a la aparición de novedosas herramientas, como el webmail, y al nacimiento de diferentes tipos de contenidos. Este movimiento gana especial notoriedad con la Web 2.0, momento en el que los mainstream media pierden el centro del 
ecosistema mediático con la aparición de la blogosfera y, más tarde, de las redes sociales online. En consecuencia, durante la primera década del siglo XXI, el ecosistema sufre un cambio radical: los medios de comunicación tradicionales pierden la centralidad del sistema y pasan a disputar audiencias con el público anteriormente conocido como audiencia (Rosen, 2008).

En este ambiente efervescente, los medios de comunicación se vieron obligados a migrar a la Web. No solo en la transposición de contenidos tradicionales, el shovelware inicial, como también mediante la oferta de nuevos productos adaptados al medio. Periódicos, radios y televisiones intentaron adaptarse al nuevo medio porque el aumento de usuarios de Internet rápidamente la definieron como un espacio preferencial para las audiencias. Con un crecimiento constante, la tasa de penetración mundial de Internet ascendió, en julio de 2020, al 62\% (Miniwatts Marketing Group, 2020), un valor apenas superado por los teléfonos móviles y muy por encima de todos los demás medios.

Si para la prensa este crecimiento supuso una amenaza a su modelo económico tradicional (venta de publicidad asociada a la venta de contenidos), para la radio supuso el inicio de una nueva y original forma de relación entre la audiencia y los contenidos (Priestman, 2002).

\section{La radio en Portugal}

Para entender el contexto se presenta el panorama de la radio portuguesa, con un encuadre a partir de los años 80 del siglo Xx. Esta opción está relacionada con dos factores: en primer lugar, porque en la etapa inicial de la radio en Portugal, la tendencia fue similar a la de los otros países, aunque con algún retraso. En segundo lugar, porque durante el Estado Novo, un régimen autoritario, el poder político limitó el desarrollo normal del medio.

Sin bien las primeras emisoras piratas datan de 1977 (Santos, 2005), el gran movimiento de las radios libres en Portugal ocurre al inicio de los años 80, que llegarán a ser alrededor de 600 (Bonixe, 2015). Sin una legislación que regulase su actividad, las autoridades perseguían a las emisoras y se apoderaban de los equipos, muchos de ellos desarrollados de forma artesanal. Sin embargo, al final de la década, la Ley 87/88, de 30 de julio (1988) vendría a regular la actividad, asignando 314 frecuencias a radios locales.

Por aquel entonces, Portugal estaba pasando por un período de resplandor económico gracias a su integración en la Unión Europea. Con la mejora del nivel de vida, las ventas de electrodomésticos se dispararon y, entre ellos, los aparatos de televisión. En un escenario adverso, en el que la televisión ganaba nuevas audiencias, la radio logró encontrar una forma de sobrevivir al nuevo medio. Las radios libres ofrecieron una multiplicidad de nuevas voces, pero 
explotaron sobre todo una fragilidad de las televisiones: la mayor complejidad técnica de este medio, que obliga a las televisiones a centrar su información en los grandes núcleos urbanos donde tenían sus redacciones. La abrumadora mayoría de las emisoras libres eran locales y la cercanía, hasta entonces apenas explotada por la prensa, permitió al medio radiofónico diferenciarse de la competencia y ganar un lugar especial en el corazón de las audiencias.

Los cambios legislativos posteriores (1997 y 2001) llevaron a una reorganización de la radio en Portugal, con la aparición de grandes grupos como RDP, Renascença y Media Capital o radios de referencia como TSF. Son grupos que van cambiando su nombre y organigrama, pero siguen siendo los más influyentes, coexistiendo en paralelo con otros pequeños grupos regionales o temáticos.

En la década de los 90, las radios iniciaron un nuevo proceso de adaptación a las novedades, en este caso la aparición de Internet. La Radio Comercial fue la primera en tener emisión en directo en la Web, lo que ocurrió en agosto de 1995 (Silva, 1999 citado en Bastos, 2015). Se han seguido varios registros de dominios, pero solo en septiembre de 1996, después de unos seis meses de pruebas, nace la TSF online (Bastos, 2000), con la disponibilidad de varios tipos de contenidos en su plataforma web y de servicios informativos en directo. TSF fue también la primera radio portuguesa en ofrecer podcast, a partir de enero de 2006 (Bastos, 2015).

En 2007, el proyecto ROLI, de la Associação Portuguesa de Radiodifusão, permitió a las estaciones de radio locales tener su transmisión en Internet y, al año siguiente, ya 195 radios locales tenían transmisión en la red (Bonixe, 2015). Con un nuevo medio en el ecosistema, las empresas de radiodifusión buscaron una vez más rentabilizar sus potencialidades de la mejor forma posible. Si para la prensa la Web fue el principio del fin del modelo económico hasta entonces usado, con la entrada en una de sus peores crisis, para la radio fue una oportunidad de mostrar su instinto de supervivencia, usando el nuevo medio para ampliar su cobertura.

Buscando cuadrar las nuevas realidades, a inicios de este siglo se aprueba una nueva Ley de la Radio (2010) para regular el acceso a la actividad y su ejercicio. Esa legislación ha sido actualizada pasados cinco años con una buena Ley (2015) destinada a promocionar la transparencia de la propiedad, la gestión y los recursos financieros de los medios de comunicación en Portugal, incluyendo la radio.

En la actualidad, el panorama radiofónico portugués presenta dos realidades: por una parte, un conjunto de grandes grupos: RTP [Antena 1 (generalista/información), Antena 2 (cultura), Antena 3 (joven) y un conjunto de webradios], Media Capital Rádios [Rádio Comercial (generalista/música), M80 
(música), Rádio Cidade (música) y webradios], R/com - Renascença Comunicação Multimédia [Rádio Renascença (generalista), RFM (música), e Mega FM (música). Existen otras radios importantes, como TSF (Grupo Global Media,) y emisoras que pertenecen a empresarios como Álvaro Covões, propietario de la promotora de espectáculos Everithing is New (Radar, Oxigénio, EIN e Nos Alive) y Luís Montez, propietario de la promotora Música no Coração, (Marginal, Festival, Nova Era, Amália, Meo Sudoeste e SBSR). En 2020, por distribución hertziana en FM existen en Portugal seis radios nacionales (Antena 1, Antena 2, Antena 3, Rádio Comercial, Rádio Renascença e RFM), dos regionales (TSF e M80) y 318 emisoras locales ${ }^{8}$.

\section{Metodología}

Al tratarse de una reflexión teórica, se optó por hacer una revisión bibliográfica sobre los procesos de cambio ocurridos en el ecosistema y un análisis de las características propias de la radio. Con esas características, se pasó a una metodología cualitativa, un estudio de caso de carácter descriptivo buscando explicar el fenómeno en su contexto (Yin, 1994), en este caso a través de la ejemplificación con programas portugueses que ilustran cómo la radio se adaptó al nuevo ecosistema mediático. Obviamente no se trata de una originalidad de la radio portuguesa: el marco propuesto - estudiar la presencia de las cuatro características identificadas- puede aplicarse a cualquier país.

\subsection{Objeto de Estudio}

Es este trabajo se buscan identificar las cuatro características de la radio online - unisensorialidad, ubicuidad, multimedialidad e interactividad- en la programación de las radios portuguesas. Aunque por audiencia total las radios locales presentan buenos números, se optó por restringir el análisis a los programas emitidos en las radios nacionales: Antena 1, Antena 2, Antena 3, Rádio Comercial, Rádio Renascença y RFM.

\subsection{Variables}

Los sentidos son elementos centrales para la traducción del mundo que nos rodea. A través de los receptores sensoriales —ojos, oídos, piel, nariz y bocanuestro cerebro recibe e interpreta señales que nos permiten tener una percepción del mundo en el que vivimos. Cuanto más sensibles y variados sean los receptores usados, mejor será la recogida de información llevada al cerebro para su consiguiente interpretación.

8 En el caso de las webradios, la emisión carece de registro, pero no de licencia. Los registros de ANACOM presentan 91 webradios en Portugal, pero existen muchas más sin registro. 
Por sus características técnicas, los medios de comunicación exigen competencias sensitivas para su consumo. En teoría se puede decir que, a un mayor número de sentidos involucrados corresponde una mayor riqueza y facilidad para la decodificación debido al estímulo de más sentidos. Es decir, cuantos más sentidos sean estimulados, más apelativo y envolvente es el medio de comunicación. ¿Pero funciona así en todas las situaciones de la realidad cotidiana? Lo veremos en la explicación de las variables.

\subsubsection{Ubicuidad}

Una de las mayores limitaciones técnicas de la radio hertziana se refiere a la distribución de la señal. En el caso específico de las emisoras locales, la cobertura se circunscribe a una zona geográfica de proximidad, pero con la Web, las radios adquirieron un alcance global. «Estar presente en Internet significa para las radios locales expandir su emisión por el mundo, lo que puede representar un conjunto de oportunidades para estas emisoras, técnicamente vinculadas a un espacio limitado cuando emiten sólo por vía hertziana» (Cordeiro \& Bonixe, 2019: 33).

En un país como Portugal, donde las migraciones hacia el litoral y a otros países tuvieron un efecto devastador en la demografía, la posibilidad de llevar la información hasta los ciudadanos de la región dispersos por el mundo se ha convertido en una enorme oportunidad para todas las radios, pero especialmente para las emisoras locales que superaron las limitaciones técnico-legales de su restricta cobertura, asentando su noción de comunidad en los intereses y estilos de vida comunes (Evans \& Smethers, 2001).

Como señala Silva (2012: 149), «technologies cannot be denied, and new paths are been traced. More than listen, we have radio's physical presence through its front door: the website» 9 . Una vez más, la potencial amenaza representada por la aparición de un nuevo medio de comunicación —la Web—se transformó en una oportunidad que ofreció a la radio la ubicuidad.

\subsubsection{Unisensorialidad}

La prensa requiere la visión, el tacto y puede también envolver al olfato una vez que el olor de la tinta es apreciado por muchos lectores. La televisión apela a la visión y a la audición, pudiendo apelar al tacto por la interacción con mandos de juegos. La Web requiere visión y audición en el consumo de los contenidos, necesitando aún el tacto siempre que ocurre interacción a través de cualquier tipo de periférico. En este escenario de multiplicidad de sentidos, el hecho de

9 «No se pueden negar las tecnologías y se trazan nuevos caminos. Más que escuchar, tenemos la presencia física de la radio a través de su puerta principal: el sitio web». 
que la radio solo requiera la audición parece ser una fragilidad, sin embargo, una de las mayores debilidades de la radio - la unisensorialidad - podría ser, al mismo tiempo, una de sus grandes ventajas.

Por un lado, porque a una menor exigencia sensitiva asociamos la simplificación del mensaje y, de esa forma, la liberación del consumidor para experimentar nuevas sensaciones. Hendy (2010) defiende que la experiencia de escuchar la radio en la oscuridad crea una especie de magia nacida de la unión entre el sonido y el tiempo. La oscuridad representa aquí la anulación de los demás sentidos, liberando al ser humano para la fruición del sonido. Como bien nota Rubio (2015: 18) la radio debe «potenciar su característica principal, el sonido, de modo que se siga llevando la señal por las ondas hertzianas y se propaguen con la tecnología por los megabytes»

Además de ser una ventaja, esta unisensorialidad libera los demás sentidos hacia otras actividades, subrayando así el papel de la radio a modo de compañía y su utilización como complemento a otras actividades, es decir, la simultaneidad con otras prácticas como pueden ser la conducción de vehículos o el desarrollo de rutinas laborales.

\subsubsection{Interactividad}

Otra área donde Internet alteró la forma de hacer radio fue en la relación con los usuarios. Desde un punto de vista secuencial, la relación entre radios y audiencias empezó a darse a través de cartas, pasó al teléfono, más tarde al correo electrónico y, por fin, a las redes sociales online. «La participación a través de Facebook y Twitter se desarrolla en tiempo real. El flujo de mensajes es permanente y sincrónico» (Rost, 2014: 65). La creciente utilización de estas redes tuvo dos efectos: por un lado, un conocimiento más profundo de los oyentes facilitado por el acceso a sus perfiles en las redes sociales. Por otro lado, la instantaneidad de la comunicación a través de la Web ha prestado más dinamismo a las emisiones en línea, mejorando así la interactividad.

\subsubsection{Multimedialidad}

No obstante, la radio no se limitó a aprovechar la Web para ampliar su zona de acción. Este es un beneficio importante, pero el medio pasó también a explotar la presencia en la Web para complementar sus contenidos sonoros con otro tipo de contenidos. La multimedialidad pasó a ser una marca de la radio en la Web, como demuestra el cambio de nombre de la Rádio Renascença: desde marzo de 2010 pasó a denominarse R/com - Renascença Comunicação Multimédia para reflejar su posicionamiento y su oferta que incluye webradios y webTVs, como $(\mathrm{V}+)$ que ganó 7 de los 11 premios nacionales de Excelencia en Ciberperiodismo. La multimedialidad es hoy una característica de la radio gracias a su 
presencia en la Web y se asume como una oportunidad para mantener e, incluso, aumentar a sus públicos.

\section{Resultados y Discusión}

En este apartado se ilustra cada una de las características de la radio anteriormente enunciadas con ejemplos de programas portugueses que confirman la manera en que la radio se adaptó al nuevo ecosistema a través de procesos de remediación inversa.

Ubiquidad: con emisiones en la Web desde 1995, pronto la radio portuguesa mostró que pretendía explorar en nuevo medio, buscando usar las herramientas de Internet como forma de sobrevivir en el ecosistema. En una primera fase, su principal preocupación ha sido extender de forma significativa su cobertura geográfica, amplificando la ubicuidad propia de Internet. Esta característica se ha convertido en una marca identitaria de cualquier radio, independiente de su dimensión o poder económico, y en actualidad está presente en prácticamente todas las emisoras portuguesas, presentándose como un buen ejemplo de la remediación inversa.

Pero la remediación inversa ocurrió también a nivel de los contenidos. Uno de los primeros programas en usar contenidos de la Web en su emisión regular fue $O$ meu blogue dava um programa de rádio: «A Rádio Comercial, primeira estação portuguesa a emitir em directo na Internet, é agora a primeira a trazer o fenómeno dos blogs para o éter. 0 que lhe propomos é transformar o seu blog numa hora de rádio. Nós pegamos nas suas palavras e juntamos a música, dando uma vida nova ao seu refúgio na Internet» (Rádio Comercial, 2006) ${ }^{10}$.

Unisensorialidad: el hecho de que el consumo de radio pueda ocurrir simultáneamente al desarrollo de otras actividades es otra ventaja de la radio tradicional proporcionada por su unisensorialidad, pero que ahora tiene nuevas opciones. Una de ellas es el recurso al podcast, rompiendo así una de las ataduras de la radio: su consumo lineal que implicaba la audición en directo. Con el podcast pasó a ser posible el consumo en diferido y en dispositivos personales y móviles, como los reproductores de MP3 y, más recientemente, los smartphones. Como bien nota Cebrián Herreros (2009: 19), el podcast es «una prolongación de la ciberradio que la convierte en portable, nómada y

10 «Radio Comercial, la primera estación portuguesa de radiodifusión con emisión en vivo por Internet, ahora es el primero en llevar el fenómeno de los blogs al éter. Lo que le proponemos es transformar su blog en una hora de radio. Nosotros tomamos sus palabras y juntamos la música, dando una vida nueva a su refugio en Internet"». La página del programa ya no está disponible. 
desconectada de la Red en el momento de la audición». Un ejemplo portugués de podcast, de los muchos que existen, es el espacio Ora Deixa Cá Ver (esse Whatsapp)!, ofrecido por Rádio Comercial y dirigido por Ana do Carmo. En su descripción se dice que «Ora Deixa Cá Ver é o podcast onde os convidados aceitam, de livre e espontânea vontade, mostrar as conversas de Whatsapp. Sim, o Whatsapp!!!»11 (Rádio Comercial, 2019). Se enfatiza este podcast porque el programa se basa en contenidos de un medio posterior a la radio, el móvil. En sentido contrario, el programa Obrigado, Internet, emitido en Antena 3 entre 2016 y 2019 (Nuno Dias, Pedro Paulos e Fernando Alvim), pasó de un podcast a emisión tradicional, con lo cual se puede ver también Internet como un laboratorio de pruebas para los programas.

Interactividad: los cambios ocurrido en este campo han venido a robustecer la instantaneidad característica de la radio gracias a la adopción de las redes sociales en casi todos los programas de animación. Siendo una presencia habitual en varios programas, se enfatiza aquí la utilización de las redes en el Programa Mañana de la M80 con el pasatiempo Acha que sabe responder. El resumen es simple: «Se é viciado no queijinho do Trivial e acha que sabe responder a tudo ou é só muito criativo nas respostas que dá, este é o momento para si: "Acha que sabe responder?". Uma pergunta por dia... que nunca fica sem resposta» (M80, 2016) ${ }^{12}$.

Otro ejemplo es el programa Não Me Obriguem a Vir para a Rua Gritar, emitido por Antena 3, cuya autoría corre a cargo de Ana Markl. Este espacio dura alrededor de 1 minuto y maneja un tema basado en comentarios de usuarios en sitios o redes sociales. «Ana Markl chafurda no lodo da indignação para levar à antena os melhores comentários daqueles que se queixam muito na internet... mas nunca irão para a rua gritar» (Antena 3, 2016) ${ }^{13}$.

Además de da interactividad basada en las redes sociales online, en actualidad presentes en casi todos los programas con participación de los usuarios, se antoja necesario destacar el proyecto Cotonete, un sitio de radios online que permitía a cada usuario hacer su propia radio y definir el estilo de música preferente. El sitio, nacido en 2001, está temporalmente fuera de servicio.

11 «Ora Deixa Cá Ver es el podcast donde los invitados aceptan, de libre y espontánea voluntad, mostrar el Whatsapp. ¡Sí, el Whatsapp!!!».

12 «Si es adicto al queso del Trivial y cree que sabe responder a todo o es sólo muy creativo en las respuestas que da, éste es el momento para usted: “¿Crees que sabes responder?”. Una pregunta por día ... que nunca se queda sin respuesta. La participación se realiza por teléfono, pero sobre todo a través de las redes sociales».

13 «Ana Markl busca en el lodo de la indignación para llevar a la antena los mejores comentarios de aquellos que se quejan mucho en internet ... pero nunca irán a la calle gritar». 
Multimedialidad: esta característica es visible en casi todos los sitios web de las radios actuales. Noticias, videoclips, o pequeños vídeos de los presentadores/invitados son ofrecidos en secciones web propias de los programas. Un ejemplo de Rádio Comercial es el Lado Sombra, un espacio donde figuras conocidas de la cultura portuguesa responden a cuestiones poco convencionales. «Vícios? Instintos assassinos? Apanhada a mentir? A prova de fogo do Rui Maria Pêgo e da Ana Martins!»14 (Rádio Comercia, 2019). Así se presenta este conjunto de vídeos que integra el programa Era o que faltava.

Se destaca el ya comentado proyecto $\mathrm{V+}$, da Rádio Renancença: al entrar en este espacio, únicamente la marca nos recuerda que se trata de un sitio de una emisora radiofónica. Las noticias en video y los reportajes rellenan un espacio que en nada se distingue del de una televisión.

\section{Conclusiones}

El hecho de que los nuevos medios se complementen y alimenten de sus predecesores justifica que haya fallado la elevada cantidad de predicciones sobre la muerte de la radio. Primero con la televisión, después con Internet, este medio vivió tiempos difíciles, pero ha sobrevivido a todas las novedades, sacando hasta partido de algunas de ellas a través de procesos de remediación inversa.

En su formato tradicional, el medio radiofónico supo potenciar su unisensorialidad, profundizando el concepto de radio compañía para acercarse a los oyentes que están desarrollando otra actividad y sólo tienen un sentido libre: la audición. Como compañera de viaje para quien conduce, trabaja o practica deporte, la radio supo igualmente libertar el usuario de las parrillas de programación al ofrecer el podcast.

En su nuevo formato online, los grupos mediáticos más fuertes se han visto favorecidos en mayor medida, pero todas las radios han ganado con Internet, independientemente de su dimensión. Los mayores beneficios se registraron a nivel de la distribución: aunque con diferentes coberturas, las radios estaban confinadas a un auditorio limitado técnicamente por las ondas hertzianas. Con la Web, la cobertura pasó a ser global siendo posible llevar la emisión a cualquier punto del mundo con conexión a Internet. Así, a través de una remediación inversa la radio adquirió una característica de la Web: la ubicuidad.

Con la presencia en la Web, la radio pasó igualmente a ofrecer contenidos de diferentes formatos, como textos, fotos y videos, entre otros. Esta

14 « ¿Adicciones? ¿Instintos asesinos? ¿Atrapado mintiendo? ¡La prueba de fuego de Rui Maria Pêgo y Ana Martins!». 
característica, la multimedialidad, es una remediación inversa de la televisión y de la Web y situó a la radio en un mismo nivel de estos medios.

Es también la Web quien ayuda a mejorar el proceso de segmentación que la radio ya había iniciado, porque pasa a ser posible multiplicar la oferta y segmentar la audiencia hasta la personalización resultante de las opciones del usuario, o de los algoritmos que leen sus hábitos de consumo.

Otro campo con fuerte influencia de la Web es en la relación con el público. El contacto por correo electrónico o redes sociales acelera y valora la interacción. Esta es la remediación inversa más visible en la radio en Portugal: tanto en la investigación de temas como en la participación, las redes sociales son una constante en programas de animación o en rúbricas.

Como destacan Barrios Rubio \& Gutiérrez García (2016: 251), «(...) las estrategias de actuación deben girar en torno a la concepción de una propuesta comunicativa bajo las nuevas dinámicas del ecosistema mediático, y la interacción y vínculo que se establece con la audiencia». La realidad portuguesa subraya que la radio tradicional se ha reconfigurado al aprovechar todas las potencialidades de los nuevos medios y, de esa forma, sobrevivió a los prematuros anuncios de su muerte inminente. Hoy, como antes, hay que contar con la radio en el nuevo ecosistema mediático.

\section{Referencias bibliográficas}

AHONEN, T. (2011). Almanac 2011. Hong Kong: TomiAhonen Consulting

ANTENA 3 (2016). Não me obriguem a vir para a rua gritar. Disponible en https://www.rtp.pt/play/p2284/e400284/nao-me-obriguem-a-virpara-a-rua-gritar

BARRIOS RUBIO, A. (2015). La radio colombiana frente al reto digital. Comunicación y Medios, 31, pp. 1-19.

\section{doi.org/10.5354/0719-1529.2015.34367}

BARRIOS RUBio, A. \& GUTIÉRREZ GARCÍA, M. (2016). Transición y adaptación de la industria radiofónica colombiana privada al entorno digital desde la perspectiva de los operadores. Revista ICONO14, 14 (2), pp. 231-255.

\section{doi.org/10.7195/ri14.v14i2.959}

BASTOS, H. (2000). Jornalismo Electrónico: Internet e Reconfiguração de práticas nas redações. Coimbra: Minerva.

BASTOS, H. (2000). Origens e evolução do Ciberjornalismo em Portugal: os primeiros vinte anos (1995-2015). Porto: Edições Afrontamento.

BolTER, J. D. \& GRUSIN, R. (1999). Remediation. Understanding New Media. Cambridge: The MIT Press.

BONIXE, L. (2015). Rádios locais em Portugal entre a proximidade e as regras do mercado. Jornalismo \& Jornalistas, 61, pp.28-32. 
CANAVILHAS, J. (2011). El nuevo ecosistema mediático. index.comunicación, 1(1), 13-24. Disponible en https: / /journals.sfu.ca/indexcomunicacion/index.php/indexcomunicacion/article/view/4/16

CANAVILHAS, J. (2012). Da remediação à convergência: um olhar sobre os media portugueses. Brazilian Journalism Research, vol. 8, 1.

Cebrián Herreros, M. (2009). Expansión De La Ciberradio. Enl@ce: Revista Venezoelana de Información, Tecnología y Conocimiento, Vol. 6, 1, pp. 11-23.

Cordeiro, P. \& BoniXe, L. (2019). Pirata, Local, Global - que radio no século XXI? Jornalismo \& Jornalistas, 68, pp.26-33.

Evans, C. J. \& Smethers, J. S. (2001). Streaming Into The Future. A Delphi Study of Broadcasters Attitudes Toward Cyber Radio Station. Journal of Radio Studies, 8: 1, pp. 5-28. doi.org/10.1207/s15506843jrs0801_4

FIDLER, R. (1997). Mediamorphosis: Understandig New Media.Thousand Oaks: Pine Forge Press.

HENDY, D. (2010). Listening in the dark: night-time radio and a "deep history" of media. In Media History, 16(2), pp.215-232.

doi.org/10.1080/13688801003656249

JeAnneney, J-N. (1996). Uma História da Comunicação Social. Lisboa: Terramar. LEI no 87/88, de 30 de julho de 1988. Assembleia da República. Diário da República n.o 175/1988, Série I de 1988-07-30. Disponible en http: / / www.dre.pt

LEI n. - 54/2010, de 24 de dezembro de 2010. Assembleia da República. Diário

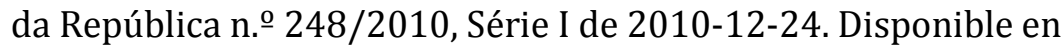
http://www.dre.pt

LEI n. - 78/2015, de 29 de julho de 2015. Assembleia da República. Diário da República n. 146/2015, Série I de 2015-07-29. Disponible en http: / /www.dre.pt

LEVINSON, P. (1997). The Soft Edge: a natural history and future of the information Revolution. London: Routledge.

M80 (2016). Acha que sabe responder. Disponible en https://m80.iol.pt/acha-que-sabe-responder/1

MCLUHAN, M. (1969). Os meios de comunicação como extensões do Homem. S. Paulo: Editora Cultrix.

MiniWATTS MARKETING GRouP (2020). Internet World Stats. Disponible en https: / / www.internetworldstats.com/stats.htm

OBCIBER (2019). Regulamento dos Prémios de Ciberjornalismo. Disponible en https://obciber.wordpress.com/premios/

PoRTEla, P. (2015). A voz do utilizador na mediamorfose da rádio: a interatividade e os consumos radiofónicos do início do século XXI. Tesis de 
Doctorado. Universidade do Minho, Braga, Portugal. Disponible en http://hdl.handle.net/1822/35828

PRIESTMAN, C. (2002). Web radio: radio production for Internet streaming, Oxford: Focal Press.

RÁDIO COMERCIAL (2006). O meu blogue dava um programa de rádio. No disponible online.

RÁDIo COMERCIAL (2019). Ora deixa cá ver. Disponible en https://podcasts.apple.com/pt/podcast/r\%C3\%A1dio-comercialora-deixa-c\%C3\%A1-ver/id1458334091

RÁDIO RENASCENÇA (2019). RR/V+. Disponible en https://rr.sapo.pt/vmais

RosT, A. (2014). Interatividade: Definições, estudos e tendências. In J. Canavilhas (Ed.), Webjornalismo: 7 caraterísticas que marcam a diferença, pp.53-88. Covilhã: Livros Labcom. Disponible en http://www.labcom-ifp.ubi.pt/ficheiros/20141204201404_webjornalismo_jcanavilhas.pdf

ROSEN, J. (2008). The people formerly known as the audience. In N. CARPENTIER, \& B. DeCleEn (EDS.), Participation and Media Production: Critical Reflections on Content Creation. Cambridge Scientific Publishers Ltd.

SANTOS, R. (2005). Rádio em Portugal: tendências e grupos de comunicação na actualidade. Comunicação e Sociedade, vol. 7, pp.137-152.

SiLVA, N. M. (2012). Radio and the Web: BBC Radio as a new model of radio communication. In MADALENA OLIVEIRA; PEDRo PORTELA; LUís ANTÓNIO SAntos (EDS.), Radio evolution: Conference Proceedings, pp.413-420. Disponible en http://www.lasics.uminho.pt/ojs/index.php/radioevolution/article/download/836/812

YIN, R. K. (1994). Pesquisa Estudo de Caso - Desenho e Métodos. Porto Alegre: Bookman. 